\title{
CONVERGENCE OF THE SPECTRAL METHOD FOR STOCHASTIC GINZBURG-LANDAU EQUATION DRIVEN BY SPACE-TIME WHITE NOISE*
}

\author{
DI $\mathrm{LIU}^{\dagger}$
}

\begin{abstract}
In this paper, a spectral method is formulated as a numerical solution for the stochastic Ginzburg-Landau equation driven by space-time white noise. The rates of pathwise convergence and convergence in expectation in Sobolev spaces are given based on the convergence rates of the spectral approximation for the stochastic convolution. The analysis can be generalized to other spectral methods for stochastic PDEs driven by additive noises, provided the regularity condition for the noises.

Keywords: spectral method, Ginzburg-Landau equation, stochastic partial differential equa-
\end{abstract} tions, space-time white noise, rate of convergence.

\section{Introduction}

In this paper, a spectral approximation is presented as a numerical method and the rates of pathwise convergence and convergence in expectation are given for the stochastic PDEs of the following form:

$$
u_{t}=-A u+R(u)+\epsilon \dot{w}(t), \quad u(0)=u_{0}, \quad t \geq 0,
$$

in a separable Hilbert space $\mathbb{H}$ equipped with the inner product $\langle\cdot, \cdot\rangle_{\mathbb{H}} \in \mathbb{R}$. $A$ is a self adjoint linear operator on domain $D(A) \subset \mathbb{H}$ with eigenvalues $0 \leq \lambda_{1} \leq \lambda_{2} \leq$ $\cdots \leq \lambda_{N} \leq \cdots, \lim _{k \rightarrow \infty} \lambda_{k}=\infty$ and a complete orthonormal system of eigenvectors $e_{1}, \cdots, e_{N}, \cdots$, such that $A e_{i}=\lambda_{i} e_{i} . R$ is a nonlinear function from $D(R) \subset \mathbb{H}$ to $\mathbb{H}$. $w(t)$ is a Wiener process with values in $\mathbb{H}$ and covariance operator $Q$.

A case of special interest is that in which $w(t)$ is the space-time white noise, i.e. $Q$ is the identity operator, which best models the fluctuations generated by microscopic effects in a homogeneous physical system. Typical situations include molecular collisions in gases and liquids, electric fluctuations in resistors, etc. ([7]) Instead of modeling each microscopic unit, which will lead to a very large complex system, people usually represent the micro effects by random perturbations in the dynamics of the macro observables. When the scale of the micro effects is very small, they should be uncorrelated jointly in time and space. After subtracting the mean intensity and putting it into the macro dynamics, we can approximate the random perturbation by the space-time white noise.

The finite difference and finite element methods for equations similar to (1.1) driven by space-time white noise have been proposed ([11], [8], [9], [4]). Uniform convergence was given for equations with Lipschitz nonlinear terms and convergence in probability was shown for equations with more general nonlinear terms. For linear equations or equations with nonlinear terms with bounded derivatives, the convergence rate of the expectation was analyzed in [2], [5] and [19]. The numerical experiments for the spectral methods for stochastic Burgers and Navier-Stokes equations were done in [16]. In this paper, a method to give the convergence rates of pathwise convergence and convergence in expectation of the spectral method for stochastic equation (1.1) driven by space-time white noise is proposed.

\footnotetext{
*Received: November 12, 2002; accepted (in revised version): January 28, 2003.

†Program in Applied and Computational Mathematics, Princeton University, Princeton, NJ 08544, USA. (dliu@princeton.edu)
} 
To illustrate our method, we choose an example equation which describes the simplest bistable system in a noisy environment. Consider the following stochastic Ginzburg-Landau (SGL) equation on $[0, L]$ driven by space-time white noise:

$$
u_{t}=\Delta u+\mu u-\lambda u^{3}+\epsilon \dot{w}, \quad u(0)=u_{0} \in \mathbb{C}_{0}[0, L], \quad t \geq 0,
$$

with fixed parameters $\mu>0$ and $\lambda>0$ and Dirichlet boundary condition:

$$
u(t, 0)=u(t, L)=0 .
$$

We chose $\left\{e_{k}(x), k \in \mathbb{N}\right\}=\left\{\frac{\sqrt{2} \sin (k \pi x / L)}{\sqrt{L}}, k \in \mathbb{N}\right\}$ to be an orthonormal basis of the Hilbert space $\mathbb{L}_{0}^{2}[0, L]$. Then

$$
\Delta e_{k}=-\lambda_{k} e_{k}, \quad \lambda_{k}=(k \pi / L)^{2} .
$$

Let $\mathbb{H}_{0}^{\alpha}=\left\{u=\sum_{k \in \mathbb{N}} u_{k} e_{k}(x),|u|_{\mathbb{H}_{0}^{\alpha}}=\sum_{k} \lambda_{k}^{\alpha}\left|u_{k}\right|^{2}<\infty\right\}$. Define the semigroup $e^{\Delta t}$, $t \geq 0$ such that for $\varphi_{0}(x), \varphi(t)=e^{\Delta t} \varphi_{0}$ is the solution of the following equation:

$$
\varphi_{t}=\Delta \varphi(t)
$$

with initial value $\varphi_{0}$ and Dirichlet boundary condition. It is known that $e^{\Delta t}, t \geq 0$ defines a continuous semigroup on $C_{0}[0, L]$ and an analytic semi-group on $\mathbb{L}_{0}^{2}[0, L]$. For the latter, we can write:

$$
e^{\Delta t} \varphi=\sum_{k} e^{-\lambda_{k} t} \varphi_{k} e_{k}, \quad \varphi=\sum_{k} \varphi_{k} e_{k} .
$$

And the following estimate holds on $\mathbb{L}_{0}^{2}[0, L]$ : for any $\alpha>0$,

$$
\left\|(-\Delta)^{\alpha} e^{\Delta t}\right\| \leq M t^{-\alpha} e^{-\delta t}, \quad t>0,
$$

for constants $M$ and $\delta$ which depend on $\alpha$.

The space-time white noise $w$ can be interpreted in the sense of distribution as

$$
w(t, x)=\sum_{k} w_{k}(t) e_{k}(x),
$$

where $w_{k}$ 's are independent standard Brownian Motions on a probability space $(\Omega, \mathcal{F}$, $\left.\mathcal{F}_{t}, \mathbb{P}, \mathbb{E}\right)$. Let

$$
\Omega_{T}=[0, T] \times[0, L], \quad T \in[0, \infty) .
$$

The stochastic convolution of $e^{\Delta t}$ and $w(t)$ is defined to be the random field $W$ on $\Omega_{T}$ :

$$
W(t, x)=\int_{0}^{t} e^{\Delta(t-s)} d w(s)=\sum_{k} e_{k}(x) \int_{0}^{t} e^{-\lambda_{k}(t-s)} d w_{k}(s) .
$$

It is known that given $\gamma \in\left(0, \frac{1}{2}\right)$, there is a version of $W(t, x)$ which is $\alpha$-Hölder continuous jointly in space and time for any $\alpha \in(0, \gamma)$. We still denote by $W(t, x)$ this modification and denote by $W(t)$ the function $W(t, \cdot) \in \mathbb{C}_{0}[0, L]$. 
A solution of equation (1.2) is defined to be the solution of the integral equation

$$
u(t)=e^{\Delta t} u_{0}+\int_{0}^{t} e^{\Delta(t-s)} F(u(s)) d s+W(t)
$$

where $F(u)=\mu u-\lambda u^{3}$. Based on the regularity of $W(t, x)$ and $u_{0}$, equation (1.5) can be discussed in a pathwise way. Define

$$
z=e^{\Delta t} u_{0}+W(t)
$$

Then for each realization of $z \in \mathbb{C}\left(\Omega_{T}\right)$, (1.5) is an integral equation with continuous coefficients. It was shown in [6] that with probability one there is a unique solution $u$ of (1.5) such that $u \in \mathbb{C}\left(\Omega_{\infty}\right)$ and $u$ depends continuously on $z$. The similar regularity for the solutions on infinite domain was given by C. R. Doering [3]. Based on this, we can extend our results in subsequent sections with little change to the case of periodic boundary conditions.

\section{The spectral approximation for the stochastic convolution}

Since the solution $u$ of equation (1.5) is a continuous function of $z$, it is natural to first define the spectral approximation to $W(t)$ and show its convergence. By the continuous dependence of $u$ on $z$, the convergence of the spectral approximation for the stochastic Ginzburg-Landau equation can be analyzed. As defined above, for any $(t, x) \in \Omega_{T}$,

$$
W(t, x)=\sum_{k} e_{k}(x) \int_{0}^{t} e^{-\lambda_{k}(t-s)} d w_{k}(s), \quad \mathbb{P}-\text { a.s. } .
$$

This means $W(t, x)$ is a Gaussian random variable for each $(t, x)$. Since $W(t, x) \in$ $\mathbb{C}\left(\Omega_{T}\right)$ a.s., we can write the following equality in the $\mathbb{L}_{0}^{2}$ sense

$$
W(t, x)=\sum_{k \in \mathbb{N}} \beta_{k}(t) e_{k}(x), \quad \beta_{k}(t)=\int_{0}^{L} W(t, x) e_{k}(x) d x
$$

Here, the integral is the Riemann integral based on the continuity of $W(t, x)$. As a limit of mean zero Gaussian random variables, $\beta_{k}(t) t \in[0, \infty]$ is also a Gaussian random variable with mean zero provided the convergence of the covariance. Let

$$
h=L / N, \quad x_{j}=j \cdot h, \quad j=1, \ldots, N-1 .
$$

Then, with probability one we have

$$
\beta_{k}(t)=\lim _{h \rightarrow 0} h \sum_{j, l} e_{k}\left(x_{j}\right) e_{l}\left(x_{j}\right) \int_{0}^{t} e^{-\lambda_{l}(t-\tau)} d w_{k}(\tau) .
$$


By the fact that $\sum_{j} e_{k}\left(x_{j}\right) e_{l}\left(x_{j}\right)=0$ if $k-l \neq 0(\bmod N)$, we have

$$
\begin{aligned}
& \mathbb{E}\left[\beta_{k}(t) \cdot \beta_{k}(s)\right] \\
= & \lim _{h \rightarrow 0} \mathbb{E}\left[h \sum_{j, l} e_{k}\left(x_{j}\right) e_{l}\left(x_{j}\right) \int_{0}^{t} e^{-\lambda_{l}(t-\tau)} d w_{k}(\tau)\right] \\
& \cdot\left[h \sum_{j, l} e_{k}\left(x_{j}\right) e_{l}\left(x_{j}\right) \int_{0}^{s} e^{-\lambda_{l}(s-\tau)} d w_{k}(\tau)\right] \\
= & \lim _{N \rightarrow \infty} \sum_{m=k+n \cdot N, n \geq 0} \frac{e^{-\lambda_{m}|t-s|}-e^{-\lambda_{m}(t+s)}}{2 \lambda_{m}}\left(\sum_{j} e_{k}^{2}\left(x_{j}\right) \cdot h\right)^{2} \\
= & \frac{e^{-\lambda_{k}|t-s|}-e^{-\lambda_{k}(t+s)}}{2 \lambda_{k}} .
\end{aligned}
$$

The above equality implies that $\beta_{k}(t)$ is an Ornstein-Uhlenbeck process with parameter $\lambda_{k}$ and initial value zero. The continuity of $\beta_{k}(t)$ is implied by the Hölder continuity of $W(t, x)$. Let $M_{k}(t)=e^{\lambda_{k} t} \beta_{k}(t)$, then

$$
\mathbb{E}\left[\left(M_{k}\left(t_{1}\right)-M_{k}\left(t_{2}\right)\right) \cdot M_{k}(s)\right]=0, \quad s \leq t_{1}, t_{2} .
$$

Define the filtration $\left(\Omega, \mathcal{F}^{k}, \mathcal{F}_{t}^{k}, \mathbb{P}, \mathbb{E}\right),(k \in \mathbb{N})$ such that $\mathcal{F}_{t}^{k}=\sigma\left\{\beta_{k}(s), s \leq t\right\}$. Since $M_{k}(t)$ is Gaussian, $(2.1)$ means $M_{k}(t)-M_{k}(s),(s \leq t)$ is independent of $\mathcal{F}_{s}^{k}$. By the fact that $M_{k}(t)$ is mean zero, we have

$$
\mathbb{E}\left(M_{k}(t) \mid \mathcal{F}_{s}^{k}\right)=\mathbb{E}\left(M_{k}(t)-M_{k}(s)+M_{k}(s) \mid \mathcal{F}_{t}^{k}\right)=M_{k}(s) .
$$

Thus, we know that $M_{k}(t)$ is a continuous martingale w.r.t. $\left(\Omega, \mathcal{F}^{k}, \mathcal{F}_{t}^{k}, \mathbb{P}, \mathbb{E}\right)$.

The spectral approximation $W_{N}(t, x)$ for the stochastic convolution $W(t, x)$ is defined to be:

$$
W_{N}(t, x)=P_{N} W(t, x)=\sum_{|k| \leq N} \beta_{k}(t) e_{k}(x) .
$$

To give the convergence rates of $W_{N}(t, x)$ to $W(t, x)$, we need the following version of Borell inequality. The proof can be found in [1].

Lemma 2.1. Let $\left\{X_{t}\right\}_{t \in[0, T]}$ be a centered Gaussian process with sample paths bounded a.s. Let $|X|=\sup _{t \in[0, T]} X_{t}$. Then $\mathbb{E}|X|<\infty$, and for all $\lambda>0$

$$
\mathbb{P}\{|| X|-\mathbb{E}| X||>\lambda\} \leq 2 \exp \left\{-\frac{1}{2} \lambda^{2} / \sigma_{T}^{2}\right\},
$$

where $\sigma_{T}^{2}=\sup _{t \in[0, T]} \mathbb{E}\left[X_{t}^{2}\right]$. By Lemma 2.1, we have for $\lambda>\mathbb{E}|X|$,

$$
\mathbb{P}\{|X|>\lambda\} \leq 2 \exp \left(-\frac{1}{2}(\lambda-\mathbb{E}|X|)^{2} / \sigma_{T}^{2}\right) .
$$

Define $\|X\|=\sup _{t \in[0, T]}\left|X_{t}\right|$, then $\mathbb{E}\|X\|>\mathbb{E}|X|$ and for $\lambda>0$,

$$
\mathbb{P}\{\|X\|>\lambda\} \leq \mathbb{P}\{|X|>\lambda\}+\mathbb{P}\{|-X|>\lambda\} .
$$

Hence, for $\lambda>\mathbb{E}\|X\|$,

$$
\mathbb{P}\{\|X\|>\lambda\} \leq 4 \exp \left(-\frac{1}{2}(\lambda-\mathbb{E}\|X\|)^{2} / \sigma_{T}^{2}\right) .
$$


To apply (2.4) to the Gaussian process $\beta_{k}(t)$, we need to estimate $\mathbb{E}\left[\sup _{0 \leq t \leq T}\left|\beta_{k}(t)\right|\right]$.

Lemma 2.2. Let $\left\|\beta_{k}\right\|=\sup _{t \in[0, T]}\left|\beta_{k}(t)\right|$, then for any $\epsilon \in(0,1)$, we have

$$
\mathbb{E}\left[\left\|\beta_{k}\right\|\right] \leq \frac{C_{\epsilon}}{k^{1-\epsilon}},
$$

where $C_{\epsilon}$ is a constant independent of $k$.

Proof. Define the functions $G(k, \lambda),(k \in \mathbb{N})$ to be

$$
G(k, \lambda)=\mathbb{P}\left\{\left\|\beta_{k}\right\|=\sup _{0 \leq t \leq T} e^{-\lambda_{k} t}\left|M_{k}(t)\right| \geq \lambda\right\} .
$$

Partitioning the interval $[0, T]$ equally into $k^{2}$ pieces, we have

$$
G(k, \lambda) \leq \sum_{0<m \leq k^{2}} \mathbb{P}\left\{\sup _{0 \leq t \leq \frac{m T}{k^{2}}}\left|M_{k}(t)\right| \geq \lambda e^{\lambda_{k} \frac{(m-1) T}{k^{2}}}\right\} .
$$

By the martingale inequality, we have the following for $p \geq \frac{1}{2}$ :

$$
\begin{aligned}
\mathbb{P}\left\{\sup _{0 \leq t \leq \frac{m T}{k^{2}}}\left|M_{k}(t)\right| \geq \lambda e^{\lambda_{k} \frac{(m-1) T}{k^{2}}}\right\} & \leq \frac{\mathbb{E}\left(\left|M_{k}\left(m T / k^{2}\right)\right|^{2 p}\right)}{\lambda^{2 p} e^{\frac{2 p \lambda_{k}(m-1) T}{k^{2}}}}=\frac{C_{p}\left(e^{\frac{2 m T \pi^{2}}{L^{2}}}-1\right)^{p}}{\left(2 \lambda_{k}\right)^{p} \cdot \lambda^{2 p} e^{\frac{2 p(m-1) T \pi^{2}}{L^{2}}}} \\
& \leq \frac{\tilde{C}_{p}}{k^{2 p} \lambda^{2 p}}
\end{aligned}
$$

This implies

$$
G(k, \lambda) \leq \frac{\tilde{C}_{p}}{k^{2(p-1)} \lambda^{2 p}}
$$

Hence, we have

$$
\begin{aligned}
\left.\mathbb{E}\left[N_{k}^{T}\right]=\int_{0}^{\infty} G(k, \lambda)\right\} d \lambda & \leq k^{\epsilon-1}+\int_{k^{1-\epsilon}}^{\infty} \frac{\tilde{C}_{p}}{k^{2(p-1)} \lambda^{2 p}} d \lambda \\
& \leq k^{\epsilon-1}+\frac{\tilde{C}}{(2 p-1) k^{2 p \epsilon-1-\epsilon}}
\end{aligned}
$$

Choosing $p$ large enough such that $\frac{1}{\epsilon}<p$, we have

$$
\mathbb{E}\left[\sup _{t \in[0, T]}\left|\beta_{k}(t)\right|\right] \leq \frac{C_{\epsilon}}{k^{1-\epsilon}},
$$

for some constant $C_{\epsilon}$.

The following proposition gives the pathwise convergence rates of $W_{N}$ to $W$.

Proposition 1. Given $r \in\left[0, \frac{1}{2}\right)$ and $A_{0}>0$. Then, for any $\alpha \in(0,1-2 r)$, there is a $\beta>0$ such that for sufficiently large $N$ and $A \geq A_{0}$ :

$$
\begin{aligned}
& \mathbb{P}\left\{\sup _{t \in[0, T]}\left|\left(1-P_{M}\right) W(t)\right|_{\mathbb{H}_{0}^{r}}^{2} \leq \frac{1}{\alpha M^{\alpha}} A^{2}, M \geq N\right\} \\
\geq & 1-C_{\beta} A^{-2\left(\left[\frac{1}{2 \beta}\right]+1\right)} \exp \left\{-c A^{2} N^{2 \beta}\right\},
\end{aligned}
$$


where $C_{\beta}$ and $c$ are positive constants independent of $A_{0}, M$ and $N$ and $\left[\frac{1}{2 \beta}\right]$ denotes the biggest integer no larger than $\frac{1}{2 \beta}$, .

Proof. By definition, we have

$$
\left|\left(1-P_{M}\right) W(t, x)\right|_{\mathbb{H}_{0}^{r}}^{2}=\sum_{k>M}\left(\lambda_{k}^{r / 2} \beta_{k}(t)\right)^{2}
$$

Define the events $A_{k}^{\gamma}\left(\frac{1}{2}<\gamma<1-r\right),(k \in \mathbb{N})$ to be

$$
A_{k}^{\gamma}=\left\{\sup _{0 \leq t \leq T}\left|\beta_{k}(t)\right| \leq \frac{A}{\lambda_{k}^{(\gamma+r) / 2}}\right\} .
$$

Choosing $\epsilon$ small enough in (2.5), we have for sufficiently large $N$ and $A \geq A_{0}$,

$$
\mathbb{E}\left[\sup _{t \in[0, T]}\left|\beta_{k}(t)\right|\right]=\frac{C_{\epsilon}}{k^{1-\epsilon}}<\frac{1}{4} \frac{A}{\lambda_{k}^{(\gamma+r) / 2}}, \quad k \geq N .
$$

Hence, by (2.4), for $N$ big enough ,

$$
\mathbb{P}\left\{A_{k}^{\gamma}\right\} \geq 1-4 \exp \left\{-\frac{A^{2}}{2} \lambda_{k}^{(1-r-\gamma)}\right\}, \quad k \geq N .
$$

Since $\frac{1}{2}<\gamma<1-r$, we have

$$
0<\alpha=2 \gamma-1<1-2 r, \quad 0<\beta=1-r-\gamma<\frac{1}{2} .
$$

We also have

$$
\sum_{k>M} \frac{1}{k^{2 \gamma}}=\sum_{k>M} \frac{1}{k^{\alpha+1}} \leq \int_{M}^{\infty} \frac{1}{x^{\alpha+1}} d x \leq \frac{1}{\alpha M^{\alpha}} .
$$

And for arbitrary constant $\bar{c}>0$, we have

$$
\begin{aligned}
\sum_{k>N} \exp \left\{-\bar{c} k^{2 \beta}\right\} & \leq \int_{N}^{\infty} \exp \left\{-\bar{c} x^{2 \beta}\right\} d x \stackrel{y=x^{2 \beta}}{=} \frac{1}{2 \beta} \int_{N^{2 \beta}}^{\infty} y^{\frac{1}{2 \beta}-1} \exp \{-\bar{c} y\} d y \\
& \leq \frac{1}{2 \beta} \int_{N^{2 \beta}}^{\infty} y^{\left[\frac{1}{2 \beta}\right]} \exp \{-\bar{c} y\} d y \\
& =\frac{1}{2 \beta} \frac{\left[\frac{1}{2 \beta}\right] !}{\bar{c}^{\left[\frac{1}{2 \beta}\right]+1}} \exp \left\{-\bar{c} N^{2 \beta}\right\}
\end{aligned}
$$

Hence

$$
\begin{aligned}
& \mathbb{P}\left\{\sup _{t \in[0, T]}\left|\left(1-P_{M}\right) W(t)\right|_{\mathbb{H}_{0}^{r}}^{2} \leq \frac{1}{\alpha M^{\alpha}} A^{2}, M \geq N\right\} \\
\geq & \mathbb{P}\left\{\bigcap_{k>N} A_{k}^{\gamma}\right\} \geq 1-\sum_{k>N} \exp \left\{-\frac{A^{2}}{2} \lambda_{k}^{\beta}\right\} \\
\geq & 1-C_{\beta} A^{-2\left(\left[\frac{1}{2 \beta}\right]+1\right)} \exp \left\{-c A^{2} N^{2 \beta}\right\},
\end{aligned}
$$

where $C_{\beta}=\frac{1}{2 \beta} \frac{\left[\frac{1}{2 \beta}\right] !}{c^{\left[\frac{1}{2 \beta}\right]+1}}$ and $c=\frac{\pi^{2}}{2 L^{2}}$. 
By the estimates of the probability in Proposition 1, we can give the following convergence in expectation.

Proposition 2. Given $r \in\left[0, \frac{1}{2}\right)$ and $1 \leq p<\infty$. Then for any $\alpha \in(0,1-2 r)$,

$$
\left[\mathbb{E} \sup _{t \in[0, T]}\left|\left(1-P_{N}\right) W(t)\right|_{\mathbb{H}_{0}^{r}}^{p}\right]^{\frac{1}{p}} \leq \frac{C}{N^{\alpha / 2}},
$$

where $C$ is a constant independent of $N$.

Proof. Let

$$
Y_{T}=\sup _{t \in[0, T]}\left|\left(1-P_{N}\right) W(t)\right|_{\mathbb{H}_{0}^{r}}
$$

By Proposition 1, we have for $A \geq A_{0}$ and $N$ big enough ,

$$
\mathbb{P}\left\{Y_{T} \geq\left(\frac{1}{\alpha N^{\alpha}}\right)^{\frac{1}{2}} A\right\} \leq C_{\beta} A^{-2\left(\left[\frac{1}{2 \beta}\right]+1\right)} \exp \left\{-c A^{2} N^{\beta}\right\} .
$$

And by definition, we have

$$
\begin{aligned}
\mathbb{E}\left[Y_{T}(t)^{p}\right] & =\int_{0}^{\infty} \mathbb{P}\left\{Y_{k}(t) \geq \lambda\right\} p \lambda^{p-1} d \lambda \\
& \leq \int_{0}^{\frac{1}{\sqrt{\alpha N^{\alpha}}}} \mathbb{P}\left\{Y_{k}(t) \geq \lambda\right\} p \lambda^{p-1} d \lambda+\int_{\frac{1}{\sqrt{\alpha N^{\alpha}}}}^{\infty} \mathbb{P}\left\{Y_{k}(t) \geq \lambda\right\} p \lambda^{p-1} d \lambda .
\end{aligned}
$$

Letting $A_{0}=1$ in $(2.6)$ and $\lambda=\frac{1}{\sqrt{\alpha N^{\alpha}}} A$, we have

$$
\begin{aligned}
\mathbb{E}\left[Y_{T}(t)^{p}\right] & \leq\left(\frac{1}{\alpha N^{\alpha}}\right)^{\frac{p}{2}}\left(1+\int_{1}^{\infty} \mathbb{P}\left\{Y_{k}(t)^{2} \geq \frac{1}{\alpha N^{\alpha}} A^{2}\right\} p A^{p-1} d A\right) \\
& \leq\left(\frac{1}{\alpha N^{\alpha}}\right)^{\frac{p}{2}}\left(1+\int_{1}^{\infty} C_{\beta} p A^{-2\left(\left[\frac{1}{2 \beta}\right]+1\right)+p-1} \exp \left\{-c A^{2} N^{2 \beta}\right\} d A\right) \\
& \leq \frac{C}{N^{\alpha p / 2}}
\end{aligned}
$$

for some constant $C$.

Borell inequality implies that $\mathbb{E}\left[\sup _{t \in[0, T]}\left|\beta_{k}(t)\right|^{2 p}\right]<\infty$. Hence, we have

Corollary 2.3. Given $r \in\left[0, \frac{1}{2}\right)$ and $1 \leq p<\infty$, then

$$
\mathbb{E} \sup _{t \in[0, T]}|W(t)|_{\mathbb{H}_{0}^{r}}^{p}<\infty
$$

\section{The spectral approximation for the SGL equation}

Let

$$
P_{N}=\left\{\varphi(x): \varphi=\sum_{k \leq N} c_{k} e_{k}, c_{k} \in \mathbb{R}\right\}
$$

Denote also by $P_{N}$ the projection from $\mathbb{L}_{0}^{2}$ onto $P_{N}$. The following lemma can be found in [18]. 
Lemma 3.1. For $1<p<\infty$, the operator $P_{N}$ from $\mathbb{L}^{p}$ to $\mathbb{L}^{p}$ is uniformly bounded, i.e. $\exists C_{p}$ s.t.

$$
\left\|P_{N}\right\| \leq C_{p}, \quad \forall N \geq 1 .
$$

The spectral approximation, or the semi-discrete Fourier-Galerkin approximation, for the SGL equation has the following form:

$$
\left[\begin{array}{l}
u_{N}(t) \in P_{N} \cap \mathbb{C}([0, T] \times[0, L]) \\
u_{N}(t)=e^{\Delta t} P_{N} u_{0}+\int_{0}^{t} e^{\Delta(t-s)} F_{N}\left(u_{N}(s)\right) d s+W_{N}(t) \\
u_{N}(0)=P_{N} u_{0}
\end{array}\right.
$$

where $F_{N}(\cdot)=P_{N} F(\cdot)$ and $F(u)=\mu u-\lambda u^{3}$. By the regularity of $W_{N}(t)$ and standard fixed point analysis, it easy to show that (3.1) has a unique continuous solution.

Taking derivatives of both sides of (3.1), we can get

$$
\left[\begin{array}{l}
u \prime_{N}(t)=\Delta u_{N}(t)+F_{N}\left(u_{N}(t)\right)-\Delta W_{N}(t)+d W_{N}(t) / d t \\
u_{N}(0)=P_{N} u_{0}
\end{array}\right.
$$

Define

$$
B_{N}(t)=W_{N}(t)-\int_{0}^{t} \Delta W_{N}(s) d s, \quad B_{N}^{k}(t)=\left\langle B_{N}(t), e_{k}\right\rangle_{\mathbb{L}^{2}} .
$$

Direct computation can show that

$$
\begin{array}{cr}
\mathbb{E}\left\{\left(B_{N}^{k}\left(t_{1}\right)-B_{N}^{k}\left(t_{2}\right)\right) \cdot B_{N}(s)\right\}=0, & s \leq t_{1}, t_{2}, \\
\mathbb{E}\left\{\left(B_{N}^{k}(t) \cdot B_{N}^{j}(t)\right)\right\}=\delta_{k j} t, & 1 \leq k, j, \leq N .
\end{array}
$$

Then by Levy's characterization of Brownian Motion, we know $B_{N}^{k}(t)$ 's are independent standard Brownian Motions. Then we give the differential form of (3.1).

$$
\left[\begin{array}{l}
u \prime_{N}(t)=\Delta u_{N}(t)+F_{N}\left(u_{N}(t)\right)+d B(t) / d t \\
u_{N}(0)=P_{N} u_{0}
\end{array}\right.
$$

The analysis of numerical numerical methods for (3.2) can be found in [15] and [14]. Since the coefficients are smooth, numerical solutions of arbitrary orders of accuracy can be given.

\subsection{A priori estimates Let}

$$
z(t, x)=e^{\Delta t} u_{0}(x)+W(t, x) .
$$

Define the integral operator on $\mathbb{L}^{q}\left(\Omega_{T}\right), 1 \leq q \leq \infty$ such that

$$
G(v)=\int_{0}^{t} e^{\Delta(t-s)} v(s) d s .
$$

Then the solution of the SGL equation is a fixed point on space $\mathbb{C}\left(\Omega_{\infty}\right)$, such that

$$
u=G(F(u))+z
$$


The following lemma shows the smoothing effect of the semi-group $e^{\Delta t}$. The proof can be found in ([6]).

Lemma 3.2. Suppose $1 \leq r<3,1 \leq p, q \leq \infty$ with

$$
\frac{1}{p}=\frac{1}{r}+\frac{1}{q}-1
$$

then $G$ is a bounded operator from $\mathbb{L}^{q}\left(\Omega_{T}\right)$ to $\mathbb{L}^{p}\left(\Omega_{T}\right)$. It is shown in ([6]) that due to the positivity of the coefficients of the nonlinear terms of the SGL equation, for some constant $C$,

$$
|u|_{\mathbb{L}^{4}} \leq C\left(|z|_{\mathbb{L}^{4}}+1\right) .
$$

By the same analysis, we can show for some constant $\tilde{C}$ independent of $N$

$$
\left|u_{N}\right|_{\mathbb{L}^{4}} \leq \tilde{C}\left(\left|z_{N}\right|_{\mathbb{L}^{4}}+1\right)
$$

where

$$
z_{N}(t, x)=W_{N}(t, x)+e^{\Delta t} P_{N} u_{0}(x) .
$$

The following Proposition gives the estimates for higher order norms.

Proposition 3. Suppose $4 \leq p<\infty$, then there exists a polynomial $P(x)$ such that with probability one,

$$
|u|_{\mathbb{L}^{p}\left(\Omega_{T}\right)} \leq P\left(|z|_{\mathbb{L}^{p}\left(\Omega_{T}\right)}\right)
$$

and

$$
\left|u_{N}\right|_{\mathbb{L}^{p}\left(\Omega_{T}\right)} \leq P\left(\left|z_{N}\right|_{\mathbb{L}^{p}\left(\Omega_{T}\right)}\right)
$$

Proof. By Lemma 3.2 and the fact that $\left|\mu x-\lambda x^{3}\right| \leq \lambda|x|^{3}+\eta$ for some constant $\eta>0$, we can get the following estimate for the solution $u$ of the SGL equation:

$$
|u|_{\mathbb{L}^{p}\left(\left(\Omega_{T}\right)\right)} \leq \bar{C}\left(|u|_{\mathbb{L}^{3 q}\left(\left(\Omega_{T}\right)\right)}^{3}+|z|_{\mathbb{L}^{p}\left(\left(\Omega_{T}\right)\right)}+1\right),
$$

where $p$ and $q$ satisfies condition (3.6) for some $r$ and $\bar{C}$ is a constant which depends on $p$ and $q$. Fix $r=\frac{8}{3}$ in (3.6), then $\frac{1}{p}=\frac{1}{q}-\frac{5}{8}$. Since $4 \leq p<\infty$, we have

$$
p>\frac{27}{5 p+8} p=\frac{27}{24} \cdot 3 q .
$$

By (3.7) and the fact that $|u|_{\mathbb{L}^{p 1}} \leq C|u|_{\mathbb{L}^{p 2}}(p 1 \leq p 2)$, using (3.12) recursively, we get (3.10). By Lemma 3.1 and (3.8), we can repeat the analysis for $u_{N}$ and obtain (3.11). Due to the uniformity of Lemma 3.1, the polynomial in (3.11) is independent of $N$. Then we can choose an appropriate $P(\cdot)$ such that both (3.10) and (3.11) are satisfied.

By the Sobolev Embedding theorem, for $2 \leq p<\infty, \mathbb{H}^{\frac{1}{2}-\frac{1}{p}} \subset \mathbb{L}^{p}$. By Corollary 2.3 we have the following corollary:

Corollary 3.3. For all $1 \leq p, q<\infty$, we have

$$
\mathbb{E}\left[|u|_{\mathbb{L}^{q}\left(\Omega_{T}\right)}^{p}\right]<\infty
$$

and

$$
\sup _{N} \mathbb{E}\left[\left|u_{N}\right|_{\mathbb{L}^{q}\left(\Omega_{T}\right)}^{p}\right]<\infty
$$


3.2. Convergence of the spectral approximation To give the order of the pathwise convergence and convergence in expectation for the spectral approximation of the SGL equation, we need the following version of Gronwall's inequality. Many inequalities of this type can be found in [10]. The proof for the version to be used can be found in [17].

Lemma 3.4. Let T, $\alpha, \beta, \nu$ be positive constants. $0<\nu<1$. Then for any continuous function $f:[0, T] \rightarrow[0, \infty)$ satisfying

$$
f(t) \leq \alpha+\beta \int_{0}^{t}(t-s)^{-\nu} f(s) d s, \quad 0 \leq t \leq T
$$

we have

$$
f(t) \leq C \alpha \cdot \exp \left\{C \beta^{\frac{1}{1-\nu} t}\right\}
$$

with a positive constant $C$ which depends on $\nu$.

Let $c>0$ be the same positive constant as in Proposition 1. We say a random variable $\xi$ is of finite moments if

$$
\mathbb{E}\left[\xi^{n}\right]<\infty, \quad \forall n \in \mathbb{N} .
$$

The following theorem gives the convergence rates for pathwise convergence and convergence in expectation of the spectral approximation for the SGL equation.

TheOREM 3.5. Given $r \in[0,1 / 2)$, for any $\alpha \in(0,1-2 r)$, there are positive constants $\beta$ and $C$ and a random variable $\xi$ such that

1. for sufficiently large $N$,

$$
\begin{aligned}
& \mathbb{P}\left\{\sup _{t \in[0, T]}\left|u(t)-u_{M}(t)\right|_{\mathbb{H}_{0}^{r}} \leq \xi\left(\left|u_{0}-u_{M}(0)\right|_{\mathbb{H}_{0}^{r}}+\frac{1}{M^{\alpha / 2}}\right), M \geq N\right\} \\
\geq & 1-C e^{-c N^{2 \beta}}
\end{aligned}
$$

2. for any $p \geq 1$,

$$
\left[\mathbb{E} \sup _{t \in[0, T]}\left|u(t)-u_{N}(t)\right|_{\mathbb{H}_{0}^{r}}^{p}\right]^{\frac{1}{p}} \leq C\left(\left|u-u_{0}\right|_{\mathbb{H}_{0}^{r}}+\frac{1}{N^{\alpha / 2}}\right) .
$$

Proof. Let $e_{M}(t)=u(t)-u_{M}(t)$, we have

$$
\begin{gathered}
e_{M}(t)=e^{\Delta t}\left(u_{0}-P_{M} u_{0}\right)+\int_{0}^{t} e^{\Delta(t-s)}\left(F(u(s))-F_{M}\left(u_{M}(s)\right)\right) d s+\left(1-P_{M}\right) W(t) \\
=e^{\Delta t} e_{M}(0)+\int_{0}^{t} e^{\Delta(t-s)}\left(F(u(s))-F_{M}(u(s))\right) d s \\
\quad+\int_{0}^{t} e^{\Delta(t-s)}\left(F_{M}(u(s))-F_{M}\left(u_{M}(s)\right)\right) d s+\left(1-P_{M}\right) W(t) \\
\stackrel{\text { def }}{=} I_{1}+I_{2}+I_{3}+I_{4} .
\end{gathered}
$$


By definition,

$$
\left|I_{1}(t)\right|_{\mathbb{H}_{0}^{r}} \leq e^{-\lambda_{M+1} t}\left|e_{M}(0)\right|_{\mathbb{H}_{0}^{r}}
$$

By the semi-group property of $e^{\Delta t}$ and (1.3), we have for $0<\gamma<1$,

$$
\begin{aligned}
\left\|(-\Delta)^{\alpha} e^{\Delta t}\left(1-P_{M}\right)\right\| & \leq\left\|(-\Delta)^{\alpha} e^{\Delta(1-\gamma) t}\right\| \cdot\left\|e^{\Delta \gamma t}\left(1-P_{M}\right)\right\| \\
& \leq M t^{-\alpha} e^{-\delta(1-\gamma) t} \cdot e^{-\lambda_{M+1} \gamma t} .
\end{aligned}
$$

Let $\kappa=\delta(1-\gamma)+\lambda_{M+1} \gamma$, we have

$$
\begin{aligned}
\left|I_{2}(t)\right|_{\mathbb{H}_{0}^{r}} & \leq \int_{0}^{t}\left|(-\Delta)^{r / 2} e^{\Delta(t-s)}\left(1-P_{M}\right) F(u(s))\right|_{\mathbb{L}^{2}} d s \\
& \leq \int_{0}^{t} M(t-s)^{-r / 2} e^{-\kappa(t-s)}|F(u(s))|_{\mathbb{L}^{2}} d s \\
& \leq M\left(\int_{0}^{\infty} \frac{e^{-2 \kappa s}}{s^{r}} d s\right)^{\frac{1}{2}}\left(\int_{0}^{T}|F(u(s))|_{\mathbb{L}^{2}}^{2} d s\right)^{\frac{1}{2}} .
\end{aligned}
$$

And we have for some constant $C_{2}^{\prime}$,

$$
\int_{0}^{\infty} \frac{e^{-2 \kappa s}}{s^{r}} d s \leq \frac{\int_{0}^{\infty} s^{-r} e^{-2 s} d s}{\kappa^{1-r}} \leq \frac{C_{2}^{\prime}}{(M+1)^{2-2 r}} .
$$

By (3.13) we have for any $p \geq 1$, there is some constant $C_{2}^{\prime \prime}$ such that

$$
\mathbb{E}\left[\left(\int_{0}^{T}|F(u(s))|_{\mathbb{L}^{2}}^{2} d s\right)^{p}\right] \leq C \mathbb{E}\left[\left(|u|_{L^{6}\left(\Omega_{T}\right)}^{6}+1\right)^{p}\right] \leq C_{2}^{\prime \prime} .
$$

This implies that there is a random variable $\bar{\xi}_{2}$ of finite moments such that

$$
\left|I_{2}(t)\right|_{\mathbb{H}_{0}^{r}} \leq \frac{\bar{\xi}_{2}}{(M+1)^{1-r}}, \text { a.s. }
$$

Thus, for any $p \geq 1$, we have some constant $\bar{C}_{2}$ such that

$$
\mathbb{E} \sup _{t \in[0, T]}\left|I_{2}(t)\right|_{\mathbb{H}_{0}^{r}}^{p} \leq \frac{\bar{C}_{2}}{(M+1)^{p(1-r)}}
$$

For $I_{3}$, let $\eta=r / 2+1 / 2$, we have

$$
\left|I_{3}(t)\right|_{\mathbb{H}_{0}^{r}} \leq \int_{0}^{t}\left|(-\Delta)^{\eta} e^{\Delta(t-s)}(-\Delta)^{-1 / 2}\left(F_{M}(u(s))-F_{M}\left(u_{M}(s)\right)\right)\right|_{\mathbb{L}^{2}} d s .
$$

Again by (1.3), we have

$$
\left\|(-\Delta)^{\eta} e^{\Delta(t-s)}\right\| \leq M^{\prime}(t-s)^{-\eta} e^{-\delta^{\prime}(t-s)} .
$$


By the Sobolev inequality, we have

$$
\begin{aligned}
& \left|(-\Delta)^{-1 / 2}\left(F_{M}(u(s))-F_{M}\left(u_{M}(s)\right)\right)\right|_{\mathbb{L}^{2}} \\
= & \sup _{|w|_{\mathbb{L}^{2}}=1}\left\langle(-\Delta)^{-1 / 2}\left(F(u(s))-F\left(u_{M}(s)\right)\right), w\right\rangle_{\mathbb{L}^{2}} \\
= & \sup _{|w|_{\mathbb{L}^{2}}=1}\left\langle F(u(s))-F\left(u_{M}(s)\right),(-\Delta)^{-1 / 2} w\right\rangle_{\mathbb{L}^{2}} \\
\leq & C^{\prime} \cdot\left|F(u(s))-F\left(u_{M}(s)\right)\right|_{\mathbb{L}^{1}} \cdot|w|_{\mathbb{L}^{2}} \\
\leq & C^{\prime \prime}\left(|u(s)|_{\mathbb{L}^{4}}^{2}+\left|u_{M}(s)\right|_{\mathbb{L}^{4}}^{2}\right) \cdot\left|e_{M}(s)\right|_{\mathbb{L}^{2}} .
\end{aligned}
$$

For $1<p<\infty$, let $\frac{1}{p}+\frac{1}{q}=1$. The Hölder inequality implies that

$$
\begin{aligned}
\left|I_{3}(t)\right|_{\mathbb{H}_{0}^{r}} \leq C^{\prime \prime \prime} & \left(\int_{0}^{t}(t-s)^{\eta} e^{-\delta^{\prime}(t-s) d s}\left(|u(s)|_{\mathbb{L}^{4}}^{2}+\left|u_{M}(s)\right|_{\mathbb{L}^{4}}^{2}\right)^{q} d s\right)^{\frac{1}{q}} \\
& \left(\int_{0}^{t}(t-s)^{-\eta} e^{-\delta^{\prime}(t-s)}\left|e_{M}(s)\right|_{\mathbb{H}_{0}^{r}}^{p} d s\right)^{\frac{1}{p}} .
\end{aligned}
$$

For $1<p^{\prime}<\frac{1}{\eta}$ and $\frac{1}{p^{\prime}}+\frac{1}{q^{\prime}}=1$, we have

$$
\begin{aligned}
& \int_{0}^{t}(t-s)^{\eta} e^{-\delta^{\prime}(t-s)}\left(|u(s)|_{\mathbb{L}^{4}}^{2}+\left|u_{M}(s)\right|_{\mathbb{L}^{4}}^{2}\right)^{q} d s \\
\leq & \left(\int_{0}^{t}(t-s)^{-p^{\prime} \eta} e^{-p^{\prime} \delta^{\prime}(t-s)} d s\right)^{\frac{1}{p^{\prime}}}\left(\int_{0}^{t}\left(|u(s)|_{\mathbb{L}^{4}}^{2}+\left|u_{M}(s)\right|_{\mathbb{L}^{4}}^{2}\right)^{q q^{\prime}} d s\right)^{\frac{1}{q^{\prime}}} \\
\leq & C_{3}^{\prime}\left(|u(s)|_{\mathbb{L}^{P}\left(\Omega_{T}\right)}^{P}+\left|u_{M}(s)\right|_{\mathbb{L}^{P}\left(\Omega_{T}\right)}^{P}+1\right)^{\frac{1}{q^{\prime}}},
\end{aligned}
$$

where $P=\max \left\{4,2 q q^{\prime}\right\}$. Again by (3.13) and (3.14), we have for some random variable $\bar{\xi}_{3}$ of finite moments such that

$$
\left|I_{3}(t)\right|_{\mathbb{H}_{0}^{r}}^{p} \leq\left(\bar{\xi}_{3}\right)^{p} \int_{0}^{t}(t-s)^{-\eta} e^{-\delta^{\prime}(t-s)}\left|e_{M}(s)\right|_{\mathbb{H}_{0}^{r}}^{p} d s
$$

Define

$$
\begin{aligned}
I(s) & =\int_{0}^{s}(s-\tau)^{-\eta} e^{-\delta^{\prime}(s-\tau)} \mathbb{E} \sup _{\mu \in[0, \tau]}\left|e_{M}(\mu)\right|_{\mathbb{H}_{0}^{r}}^{p} d \tau \\
& =\int_{0}^{s} \tau^{-\eta} e^{-\delta^{\prime} \tau} \mathbb{E} \sup _{\mu \in[0, s-\tau]}\left|e_{M}(\mu)\right|_{\mathbb{H}_{0}^{r}}^{p} d \tau,
\end{aligned}
$$

then $I(s)$ is monotonically increasing. Hence, we have

$$
\begin{aligned}
\mathbb{E} \sup _{s \in[0, t]}\left|I_{3}(s)\right|_{\mathbb{H}_{0}^{r}}^{p} & \leq \bar{C}_{3} \sup _{s \in[0, t]} \int_{0}^{s}(s-\tau)^{-\eta} e^{-\delta^{\prime}(s-\tau)} \mathbb{E} \sup _{\mu \in[0, \tau]}\left|e_{M}(\mu)\right|_{\mathbb{H}_{0}^{r}}^{p} d \tau \\
& \leq \bar{C}_{3} \int_{0}^{t}(t-s)^{-\eta} e^{-\delta^{\prime}(t-s)} \mathbb{E} \sup _{\tau \in[0, s]}\left|e_{M}(\tau)\right|_{\mathbb{H}_{0}^{r}}^{p} d s .
\end{aligned}
$$

By Proposition 1 and Proposition 2, for any $\alpha \in(0,1-2 r)$ and $N$ large enough, there is a $\beta$ such that

$$
\mathbb{P}\left\{\sup _{t \in[0, T]}\left|I_{4}(t)\right|_{\mathbb{H}_{0}^{r}} \leq \frac{1}{\sqrt{\alpha} M^{\alpha / 2}}, M \geq N\right\} \geq 1-C_{\beta} \exp \left\{-c N^{2 \beta}\right\},
$$


and

$$
\left[\mathbb{E} \sup _{t \in[0, T]}\left|I_{4}(t)\right|_{\mathbb{H}_{0}^{r}}^{p}\right]^{\frac{1}{p}} \leq \frac{C}{N^{\alpha / 2}} .
$$

Thus, with probability no less than $1-C_{\beta} \exp \left\{-c N^{2 \beta}\right\}$, for $M \geq N$,

$$
\begin{aligned}
\left|e_{M}(t)\right|_{\mathbb{H}_{0}^{r}} \leq & e^{-\lambda_{M+1} t}\left|e_{M}(0)\right|_{\mathbb{H}_{0}^{r}}+\frac{\bar{\xi}_{2}}{(M+1)^{1-r}}+\frac{1}{\sqrt{\alpha} M^{\alpha / 2}} \\
& +\bar{\xi}_{3} \int_{0}^{t}(t-s)^{-\eta} e^{-\delta^{\prime}(t-s)}\left|e_{M}(s)\right|_{\mathbb{H}_{0}^{r}} d s
\end{aligned}
$$

And

$$
\begin{aligned}
\mathbb{E} \sup _{s \in[0, t]}\left|e_{N}(s)\right|_{\mathbb{H}_{0}^{r}}^{p} \leq 3^{p-1} \mid & \left.e_{M}(0)\right|_{\mathbb{H}_{0}^{r}} ^{p}+3^{p-1}\left(\frac{\tilde{C}_{2}}{(N+1)^{p(1-r)}}+\frac{C^{p}}{N^{\alpha p / 2}}\right) \\
& +3^{p-1} \tilde{C}_{3} \int_{0}^{t}(t-s)^{-\eta} e^{-\delta^{\prime}(t-s)} \mathbb{E} \sup _{\tau \in[0, s]}\left|e_{N}(\tau)\right|_{\mathbb{H}_{0}^{r}}^{p} d s .
\end{aligned}
$$

By Lemma 3.4, we have with probability no less than $1-C_{2} \exp \left\{-c N^{2 \beta}\right\}$, for some random variable $\xi$,

$$
\left|e_{M}(t)\right|_{\mathbb{H}_{0}^{r}} \leq \xi\left(\left|e_{M}(0)\right|_{\mathbb{H}_{0}^{r}}+\frac{1}{M^{\alpha / 2}}\right) \quad M \geq N .
$$

And for some constant $C^{\prime}$,

$$
\mathbb{E} \sup _{t \in[0, T]}\left|e_{N}(t)\right|_{\mathbb{H}_{0}^{r}}^{p} \leq C^{\prime}\left(\left|e_{N}(0)\right|_{\mathbb{H}_{0}^{r}}^{p}+\frac{1}{N^{\alpha p / 2}}\right)
$$

Hence

$$
\left[\mathbb{E} \sup _{t \in[0, T]}\left|e_{N}(t)\right|_{\mathbb{H}_{0}^{r}}^{p}\right]^{\frac{1}{p}} \leq C\left(\left|e_{N}(0)\right|_{\mathbb{H}_{0}^{r}}+\frac{1}{N^{\alpha / 2}}\right) .
$$

By the Sobolev Embedding theorem, we have the following corollary:

Corollary 3.6. Given $q \in[2, \infty)$, let $r=\frac{1}{2}-\frac{1}{q}$. Then for any $\alpha \in(0,2 / q)$, there are positive constants $\beta$ and $C$ and a random variable $\xi$ such that

1. for sufficiently large $N$,

$$
\begin{aligned}
& \mathbb{P}\left\{\sup _{t \in[0, T]}\left|u(t)-u_{M}(t)\right|_{\mathbb{L}^{q}} \leq \xi\left(\left|u_{0}-u_{M}(0)\right|_{\mathbb{H}_{0}^{r}}+\frac{1}{M^{\alpha / 2}}\right), M \geq N\right\} \\
\geq & 1-C e^{-c N^{2 \beta}},
\end{aligned}
$$

2. for any $p \geq 1$

$$
\left[\mathbb{E} \sup _{t \in[0, T]}\left|u(t)-u_{N}(t)\right|_{\mathbb{L}^{q}}^{p}\right]^{\frac{1}{p}} \leq C\left(\left|u-u_{0}\right|_{\mathbb{H}_{0}^{r}}+\frac{1}{N^{\alpha / 2}}\right) .
$$




\section{Generalizations}

Based on the spectral representation of the finite difference method, we believe the analysis here can be extended to the finite difference method for the SGL equation to show its convergence. To give the convergence rate, we need the regularity more than continuity. This can be done by giving the Hölder continuity of the solution $u$ through the Hölder continuity of the heat kernel and the stochastic convolution.

The same method we have developed for the SGL equation can be generalized to general stochastic PDEs with additive noise. We rewrite (1.1) in the integral form:

$$
u(t)=e^{-A t} u_{0}+\int_{0}^{t} e^{-A(t-s)} F(u(s)) d s+\int_{0}^{t} e^{-A(t-s)} d w(s) .
$$

Let $z=e^{-A t} u_{0}+\int_{0}^{t} e^{-A(t-s)} d w(s)$. Then (4.1) becomes

$$
u(t)=\int_{0}^{t} e^{-A(t-s)} F(u(s)) d s+z(t) .
$$

Hence, we can discuss the equation as a pathwise integral equation. The same approximation in section 2 for the stochastic convolution can be used for more general noise and the convergence rate can be estimated similarly. We can define the spectral approximation for (4.1):

$$
\left[\begin{array}{l}
u_{N}(t) \in P_{N} \mathbb{H} \\
u_{N}(t)=e^{-A t} P_{N} u_{0}+\int_{0}^{t} e^{-A(t-s)} F_{N}\left(u_{N}(s)\right) d s+W_{N}(t) \\
u_{N}(0)=P_{N} u_{0}
\end{array}\right.
$$

where $F_{N}(\cdot)=P_{N} F(\cdot)$ and $P_{N}$ is the projection operator from $\mathbb{H}$ to the space spanned by the first $\mathrm{N}$ eigenvalues of $A$.

The interest here is to extend our convergence result to a variety of equations (e.g. Navier-Stokes, Burgers) and more computationally efficient spectral methods (eg. Fourier-Collocation, Legendre, Chebyshev). Since the equation is pathwise defined, we can use the same technique to show the convergence and give the convergence rate of the approximation for the stochastic convolution and hence the convergence of the approximation for the equation.

The difficulty lies in the regularity of the solutions. Usually, the regularity of the solution $u$ of (4.1) depends on the regularity of the noise $w$. Smoother noises imply smoother solutions. Although considered to be the most physical, the space-time white noise sometimes reduces the regularity of the solution into a very unsatisfactory situation compared with the original unperturbed PDEs. For example, consider the following stochastic Ginzburg-Landau equation in two dimension with Dirichlet boundary condition and space-time noise:

$$
u_{t}=\Delta u-u+: u^{3}:+\dot{w} .
$$

Here, to make sense out of the equation, we have to replace $u^{3}$ by the wick ordering $: u^{3}:$ (see [12] for definition). It can be shown ([12]) that $u \in \mathbb{H}^{-1}$. On the other hand, the convergence of the spectral methods needs sufficient regularity of the solutions. For example, for the Fourier collocation $P_{c} u$ of $u \in \mathbb{H}^{m}, m>1 / 2$ we have

$$
\left|u-P_{c} u\right|_{\mathbb{H}_{0}^{r}} \leq C N^{r-m}|u|_{\mathbb{H}^{m}} .
$$

The requirement $m>1 / 2$ is not satisfied by the SGL equation here and most other interesting cases of stochastic PDEs. Hence, future work such as new efficient numerical methods or new interpretation of the equations for stochastic PDEs needs to be done. 
Acknowledgment. The author wants to thank Weinan E for all his support and advice. The work is supported by ONR grant No. N00014-01-1-0674. The author also wants to thank Qiang Du and Philip J. Holmes for their stimulating suggestions on improving this paper.

\section{REFERENCES}

[1] R. Adler, An introduction to continuity, extrema, and related topics for general Gaussian processes. Lect. Math. Statistics., Vol. 12, Inst. Math Statistics., 1990.

[2] E.J. Allen, S.J. Novosel, and Z.M Zhang, Finite element and difference approximation of some linear stochastic partial differential equations. Stochastics and Stochastics Reports, 64:117$142,1998$.

[3] C.R. Doering, Nonlinear parabolic stochastic differential equations with additive colored noise on $R^{d} \times R_{+}$: a regulated stochastic quantization. Comm. Math. Phys., 4(109):537-561, 1987.

[4] A.M. Davie and J.G. Gaines, Convergence of numerical schemes for the solution of parabolic stochastic partial differential equations. Math. Comput, 233(70):121-134, 2000.

[5] Q. Du and T.Y Zhang, Numerical approximation of some linear stochastic partial differential equations driven by special additive noises. to appear in SIAM Numer. Anal..

[6] W.G. Faris and G. Jona-Lasinio, Large fluctuation for a nonlinear heat equation with noise. J. Phys., Math. Gen., 15(A):3025-3055, 1982.

[7] C.W. Gardiner, Handbooks of Stochastic Methods for Physics, Chemistry and Natural Sciences. Springer Series in Synergetics 13, Springer-Verlag, 1983.

[8] I. Gyöngy, Lattice approximation for stochastic quasi-linear parabolic partial differential equations driven by space-time white noise I. Potential Analysis, 9:1-25, 1998.

[9] I. Gyöngy, Lattice approximation for stochastic quasi-linear parabolic partial differential equations driven by space-time white noise I. Potential Analysis, 11:1-37, 1999.

[10] D. Henry, Geometric Theory of Semilinear Parabolic Equations. Lecture Notes in Math., 840, Springer-Verlag, Berlin, 1981.

[11] G. Jetschke, Lattice Approximation of A Nonlinear Stochastic Partial Differential Equation with White Noise. International Series of Numerical Mathematics, Birkhäuser Verlag Basel, Vol. 102, 1991

[12] G. Jona-Lasinio and P.K. Mitter On the stochastic Quantization of field theory. Commun. Math. Phys., 101:409-436, 1985.

[13] I. Karatzas and S. Shreve, Brownian Motion and Stochastic Calculus. Second edition, SpringerVerlag, 1991.

[14] P.E. Kloeden and E. Platen, Numerical Solutions of Stochastic Differential Equations. Applications of Mathematics series, Springer-Verlag, Heidelberg, Vol. 23, 1992.

[15] G. Milstein, Numerical Integration of Stochastic Differential Equations. Kluwer, Dordrecht, 1995.

[16] L. Machiels and M.O. Deville, Numerical simulation of randomly forced turbulent flows, Jour. of Comp. Phys., 145:246-279, 1998.

[17] H. Okamoto, On the semi-discrete finite element approximation for the nonstationary NavierStokes equation. Journal of the Faculty of Science, Univ. of Tokyo, Sec. IA, 3(29):613-652, 1982.

[18] A. Zygmund, Trigonometrical Series. Dover Publication, 1935.

[19] Hyek Yoo, Semi-discretization of stochastic partial differential equations on $\mathbb{R}^{1}$ by a finitedifference method. Mathematics of Computation, 69:653-666, 2000. 